\title{
New facilities for astronomy education and popularization in Guanajuato, Mexico
}

\author{
Hector Bravo-Alfaro ${ }^{1}$ \\ ${ }^{1}$ Departamento de Astronomía, Universidad de Guanajuato. C.P. 36000, Mexico \\ email: hector@astro.ugto.mx
}

\begin{abstract}
With relatively low funds, the Departamento de Astronomía de la Universidad de Guanajuato, in Mexico, has successfully carried out the modernization of a rustic astronomical observatory. At this site, once it is fully refurbished, it is planned to hold not only public observations, but also to have an ambitious programme of science popularization devoted to scholars.
\end{abstract}

Keywords. Instrumentation, University of Guanajuato, Mexico

\section{Introduction}

In 1967 it was officially opened the Observatorio Astronomico as part of University of Guanajuato, about $400 \mathrm{~km}$ NW of Mexico City (see Fig. 1a). Miguel Izaguirre, an Engineer in topography, was in charge of the site, equipped at that time with two refractor telescopes of short apertures. A dome and a hall for some 40-50 people were built on the roof of the University Central Building (Fig. 1b), after what the Observatory became -unofficially- known as "La Azotea" (the roof). The hall was used by Izaguirre, for more than ten years, for teaching astronomy for engineers.
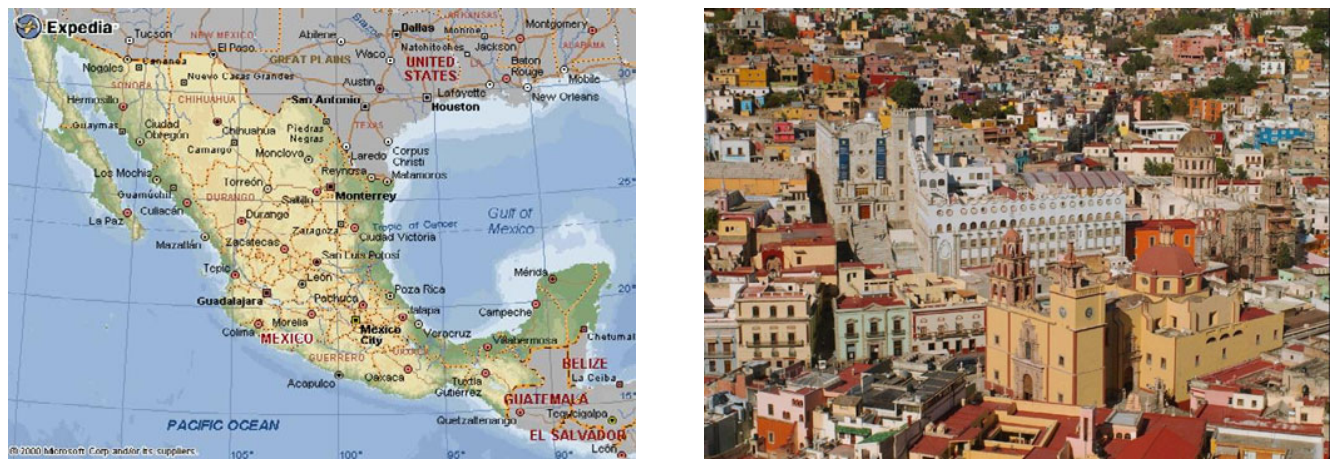

Figure 1. (a) Guanajuato City is located $60 \mathrm{~km}$ E of León, in the very centre of Mexico. (b) View of the centre of Guanajuato City; the white building in the centre corresponds to the Universidad de Guanajuato, and the dome is visible on top of it (courtesy of K.-P. Schroeder).

In the early 1980s, Miguel Izaguirre and his two sons went for a more ambitious project: a professional observatory to be installed on the hills surrounding Guanajuato City. By a fruitful collaboration between Universidad de Guanajuato and Universidad Nacional Autónoma de México (UNAM), a 57-cm Ritchey-Chrétien was installed some $25 \mathrm{~km} \mathrm{~W}$ of Guanajuato City (Fig. 2a). Years later, in 1994, these efforts gave rise to the Departamento de Astronomía (DA), where nowadays nine professional astronomers do research and teaching at both undergraduate and graduate level (http://www.astro.ugto.mx). 
However by the time Izaguirre retired, in the early 1990s, the public observatory "La Azotea" got no more maintenance nor a programme of modernization. By 2001, when the site was formally placed under the DA administration, only a portable 8-inch Schmidt existed as useful equipment. Since then, we applied a programme of free astronomical observations for non-specialized audience, but it remained to make big reparations in the building and dome, and acquire new telescopes and the necessary furniture to take advantage of the hall as conference room (Fig. 2b).
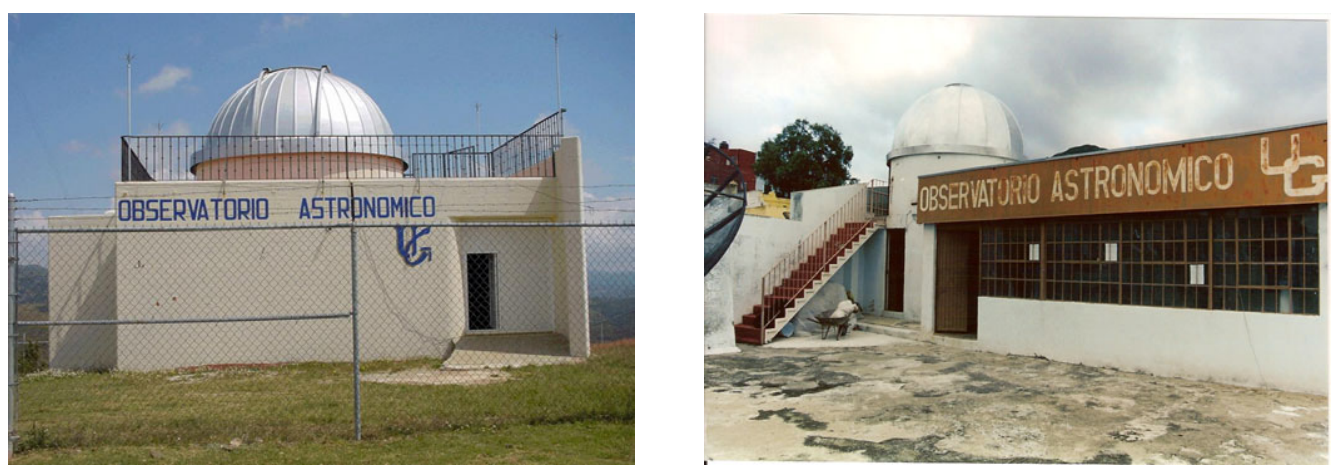

Figure 2. (a) The 0.57-m at La Luz hill. (b) La Azotea Observatory in August 2006, before the reparations.

\section{The Project}

In October 2005 the author of this work submitted a proposal to the local Council of Science (Consejo Nacional de Ciencia y Tecnologia del Estado de Guanajuato (CONACyTEG)), to get the necessary funds to acquire new equipment and furniture. The equivalent of some $\$ 30,000$ U.S. was allocated to "La Azotea" project. In addition, the University collaborated with the equivalent of $\$ 20,000$ U.S. to fully repair the building. The needed work started in September 2006 and was finished a couple of months later. At the same time, new telescopes, computing and furniture have been acquired with the aim of developing a Centre for Popularization of Science. So far the Observatory is equipped with a Schmidt Celestron 14", two Celestar 8", and a Celestron (104-mm) refractor, most of them with the necessary filters and screens to carry solar observations too. Finally, on November 22nd, the official reopening of the Observatory "La Azotea" took place. Two views of the site after reparations are shown in Fig. 3.

Once the last details are over, in January 2007, we plan to carry the following activities:

(1) A permanent programme of astronomical observations for a wide audience: in the last years, and even in the former conditions, La Azotea hosted a few hundred visitors per year. This number should naturally increase after the current upgrade.

(2) Regular conference series in different science domains: the Astronomy Department of U.G. organizes a series of five public talks on astronomy, twice per year (one in spring and one in autumn) since 1995. These conferences will now be held at La Azotea, starting in April 2007, proposing astronomical observations at the end of each talk.

(3) By a collaboration with the local department of culture we plan to host scholar groups, three times per week, for astronomical and scientific activities adapted for students between ages 6 and 15. Once this programme is applied the estimated number of students visiting the Observatory might rise above 3000 per year! 

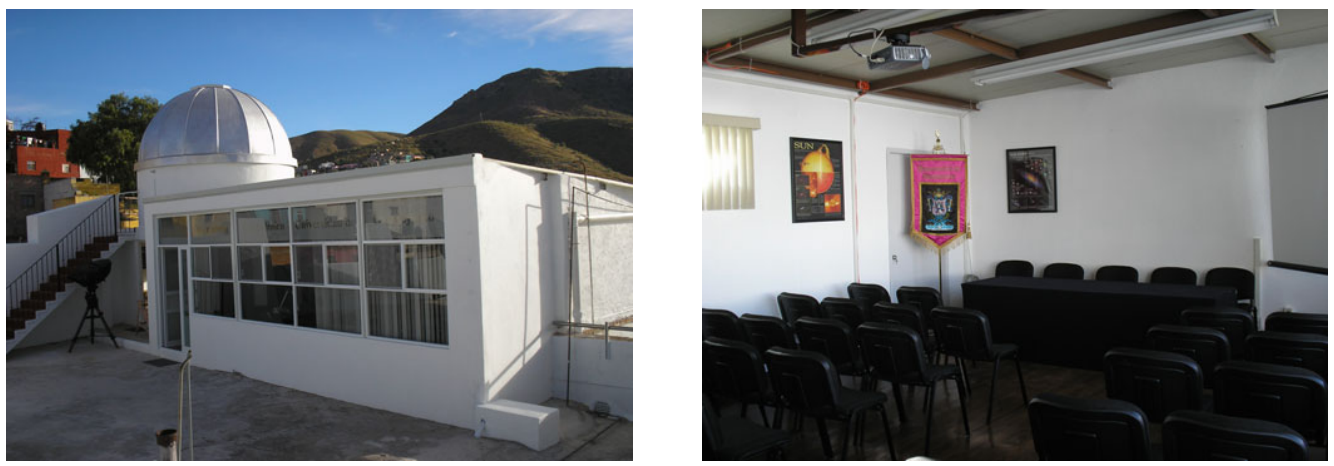

Figure 3. (a) The Observatory after the main reparations were accomplished. (b) View of the room after works and refurbishment.

(4) Summer schools in sciences for elementary and high-school teachers. In July 2007 we will carry a "pilot" school, for no more than 20 teachers, in domains such as astronomy, mathematics, physics and biochemistry.

(5) Foundation of a society of amateur astronomers.

\section{The network}

This observatory will constitute the core of the Astronomy Department's multiple facilities for wide-public astronomical observations. Two other sites, also under the DA's administration are currently at work in the Guanajuato environs: First, the professional observatory at La Luz (see Figure 2a), $25 \mathrm{~km}$ west of Guanajuato City, with a 0.57-m Ritchey-Chrétien, fully equipped with two CCDs for imaging (STL-11000, $4018 \times 2743$ pixels and STIO $2184 \times 1472$ pixels) and low resolution spectroscopy. It was recently acquired a seven-foot diameter radio telescope capable of continuum and spectral line observations in the L-band $(1.42 \mathrm{GHz})$. All these facilities are mainly devoted to training both, undergraduate and graduate students, but wide public is also host there, every week, between September and April every year since 1999. A third site is currently installed on the roof of the Astronomy Department headquarters, $3 \mathrm{~km}$ north of Guanajuato centre, where an optical 0.4-m Dobsonian and a 104-mm refractor are already available. At the moment four astronomers, a Ph.D. student and a part-time engineer for support are more concerned on popularization tasks, working several days per week on one of the different sites.

\section{Conclusions}

The Astronomy Department of U.G. is in charge of the astronomy branch of the undergraduate programme of physics, and of a graduate (M.Sc. and Ph.D.) programme in Astrophysics which started in summer 2004. We are involving students from these programs in popularization activities in order to do part of their observational training with the help of these facilities. Students in advanced levels of the undergraduate programme will participate in the activities of this Centre as part of their social duties. We expect that "La Azotea" became a centre of science popularization with important impact on students from basic levels, not only in Guanajuato (a city with more than 100000 inhabitants), but also in the environs, where much bigger cities are nearby located.

\section{Acknowledgements}

We would like to acknowledge the CONCyTEG for providing most of the funds of this project, including the fees to participate in the XXVI-GA of the IAU. 


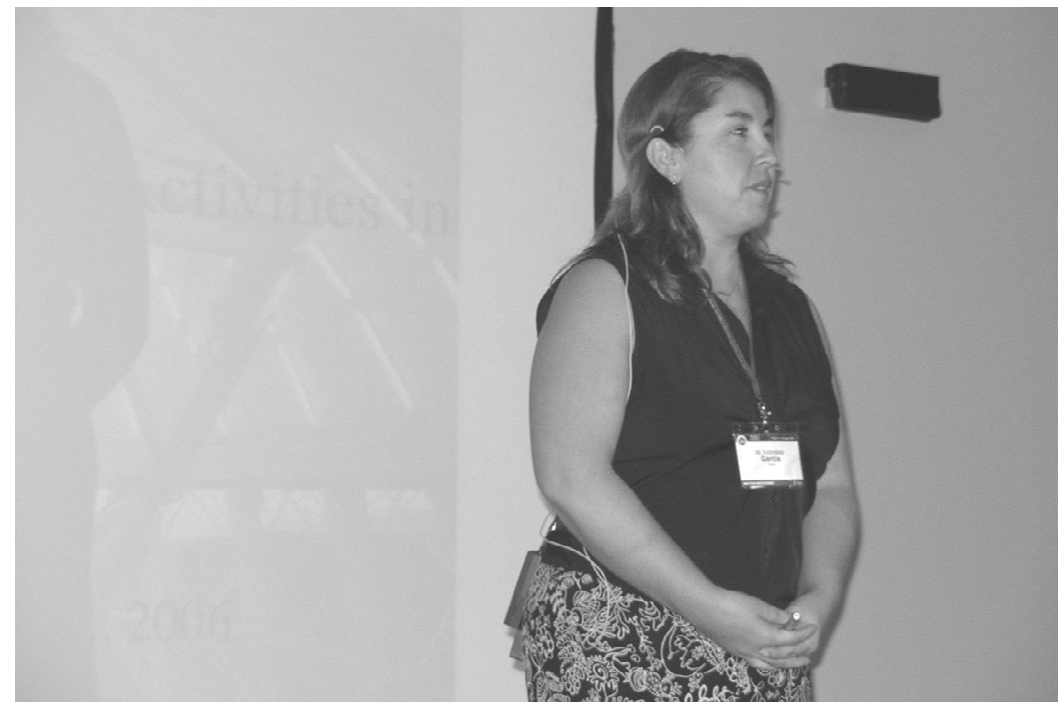

Antonieta García

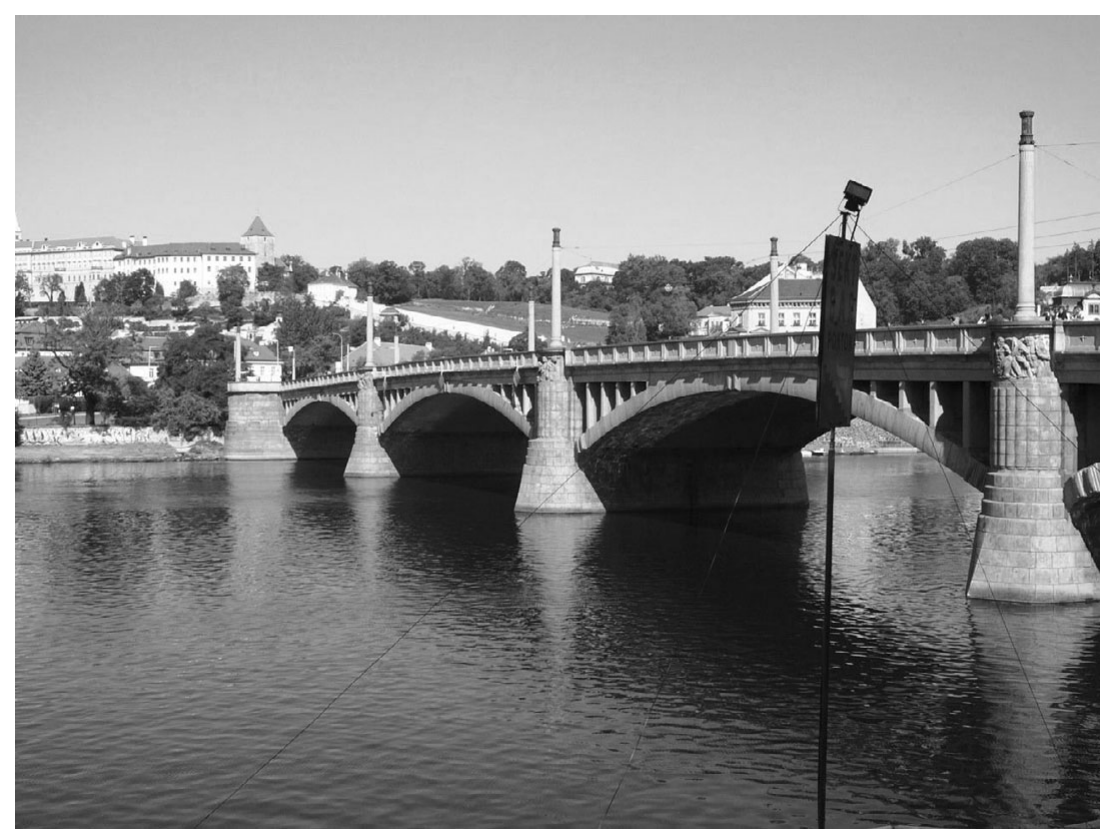

The Charles Bridge, Prague 